Revue internationale P.M.E.

Économie et gestion de la petite et moyenne entreprise

\title{
L'effet de la congruence informationnelle sur la performance financière des petites banques américaines
}

\section{Mohamed El Louadi}

Volume 8, numéro 1, 1995

URI : https://id.erudit.org/iderudit/1008274ar

DOI : https://doi.org/10.7202/1008274ar

Aller au sommaire du numéro

Éditeur(s)

Presses de l’Université du Québec

ISSN

0776-5436 (imprimé)

1918-9699 (numérique)

Découvrir la revue

Citer cet article

El Louadi, M. (1995). L'effet de la congruence informationnelle sur la performance financière des petites banques américaines. Revue internationale P.M.E., 8(1), 31-56. https://doi.org/10.7202/1008274ar
Résumé de l'article

Cet article traite des relations entre l'incertitude environnementale perçue, les degrés de complexité et de compétence technologiques, les besoins en informations, la disponibilité de ces informations et la performance financière d'un échantillon composé de 190 banques commerciales de 300 employés ou moins.

Les résultats démontrent que des liens existent entre l'incertitude environnementale perçue et les besoins en informations des chefs d'entreprise, entre la sophistication technologique et la compétence informatique perçue, d'une part, et la quantité d'informations disponibles, d'autre part. La congruence informationnelle a été calculée à partir des deux variables de besoins et de disponibilité informationnels. La relation entre la congruence et certains indicateurs de performance financière n’a pu être détectée qu'à la suite d'une analyse typologique effectuée sur l'échantillon. Cette relation n'a été observée que dans le cas de banques qui sont financièrement moins performantes que leurs concurrentes.

Notre conclusion est que l'analyse des corrélations est limitée quand elle est appliquée à un échantillon qui n'est pas homogène, puisque certaines relations peuvent être dissimulées et ne peuvent être détectées que si cet échantillon est rendu plus homogène sur la base d'une ou de plusieurs variables critères.
Ce document est protégé par la loi sur le droit d'auteur. L'utilisation des services d'Érudit (y compris la reproduction) est assujettie à sa politique d'utilisation que vous pouvez consulter en ligne.

https://apropos.erudit.org/fr/usagers/politique-dutilisation/ 


\title{
L'effet de la congruence informationnelle sur la performance financière des petites banques américaines
}

\author{
Mohamed EL LOUADI* \\ Université Concordia
}

MOTS CLÉS

\author{
Incertitude - Information - Analyse typologique \\ Besoins informationnels - Disponibilité informationnelle \\ Performance organisationnelle - Banques
}

\begin{abstract}
RÉSUMÉ
Cet article traite des relations entre l'incertitude environnementale perçue, les degrés de complexité et de compétence technologiques, les besoins en informations, la disponibilité de ces informations et la performance financière d'un échantillon composé de 190 banques commerciales de 300 employés ou moins.

Les résultats démontrent que des liens existent entre l'incertitude environnementale perçue et les besoins en informations des chefs d'entreprise, entre la sophistication technologique et la compétence informatique perçue, d'une part, et la quantité d'informations disponibles, d'autre part. La congruence informationnelle a été calculée à partir des deux variables de besoins et de disponibilité informationnels. La relation entre la congruence et certains indicateurs de performance financière n'a pu être détectée qu'à la suite d'une analyse typologique effectuée sur l'échantillon. Cette relation n'a été observée que dans le cas de banques qui sont financièrement moins performantes que leurs concurrentes.
\end{abstract}

* Mohamed El Louadi est professeur à l'Université Concordia de Montréal. Il est titulaire d'un Ph.D. en systèmes d'information (MIS) de l'Université de Pittsburgh (É.-U.). Il a publié dans des revues telles que la Revue internationale en gestion et management de projets, la Revue canadienne des sciences de l'administration, Accounting, Management and Information Technologies et Information Resources Management Journal. Membre du GREPME, il a également participé à des conférences sur les PME, notamment le Congrès international francophone de la PME tenu à Tunis en 1993 et la conférence annuelle du Conseil canadien de la PME et de l'entrepreneuriat tenue à Winnipeg en 1994. Ses intérêts de recherche portent surtout sur la gestion de l'information et des technologies de l'information dans un contexte PME. Adresse : Département des sciences de la décision et des systèmes d'information, Université Concordia, 1455, boulevard de Maisonneuve Ouest, Montréal (Québec) H3G 1M8. 
Notre conclusion est que l'analyse des corrélations est limitée quand elle est appliquée à un échantillon qui n'est pas homogène, puisque certaines relations peuvent être dissimulées et ne peuvent être détectées que si cet échantillon est rendu plus homogène sur la base d'une ou de plusieurs variables critères.

\section{ABSTRACT}

This paper addresses questions regarding the relationships between perceived environmental uncertainty, organizational technological sophistication and competence, and information requirements and availability using a sample of 190 small banks of 300 employees or less.

The results show that relationships seem to exist between perceived environmental uncertainty and chief executive officers information requirements, between organizational technological sophistication and technological competence on the one hand, and information availability on the other. Informational congruence was computed from the amount of information requirements and the amount of information availability variables. The relationship between this congruence and some financial performance indicators is established in the case of low performing banks. The distinction between the high-and the lowperforming banks was made following a cluster analysis which was performed on the data.

Our conclusion is that correlational analyses stand limited when they are applied on a heterogeneous sample since some relationships can be obscured as a result of this heterogeneity. Our study suggests that cluster analysis can be helpful in unveiling this type of relationships.

\section{RESUMEN}

Este articulo examina las relaciones entre la incertidumbre percibida del medio ambiente, el grado de sofisticación y de capacidades tecnológicas, las necesidades de información, la disponibilidad de esas informaciones y los resultados financieros de una muestra compuesta de 190 bancos comerciales de menos de 300 empleados.

Los resultados mostraron que existen vínculos entre la incertidumbre percibida en el medio ambiente y las necesidades de información de los jefes de empresa; entre la sofisticación tecnológica y la capacidad informática percibida de un lado y la cantidad de información por. La "congruencia informativa " fue calculada a partir de dos variables de necesidades y de disponibilidad informativa. La relación entre la congruencia y ciertos indicadores de desempeño financiero solo pudo ser detectada después de un análisis por grupos sobre la muestra. Esta relación solo pudo ser observada en el caso de bancos que tienen resultados financiera mente inferiores a los de sus competidores.

Nuestra conclusión es que el análisis de correlaciones es limitado cuando se aplica a una muestra que no es homogénea debido a que ciertas relaciones pueden ser disimuladas y solo pueden ser detectadas si se homogeneiza la muestra sobre la base de una o varias variables. 


\section{Introduction}

L'industrie bancaire en Amérique du Nord passe par une période de grands bouleversements économiques et technologiques. Ainsi, la déréglementation aux États-Unis a permis aux banques d'offrir un nombre croissant de nouveaux services et produits tels que la gestion de portefeuille, le courtage de valeurs, la planification financière, l'assurance-vie, les fonds de placement, les services fiduciaires, etc.

De plus, les banques nord-américaines font face à une vive concurrence internationale de la part des Japonais, des Britanniques et des Français. En 1993, le classement international de la British Credit Rating Agency IBCA, Ltd. révélait que les sept banques les plus importantes en termes d'actifs étaient japonaises. La première banque nord-américaine y est classée vingtdeuxième, et aucune banque canadienne ne figure dans la liste des 50 premières banques. Ce n'est que sur le plan des profits que la Nation Bank se classe deuxième (Glasgall et Javetski, 1993). La concurrence locale ou régionale fait que les banques s'efforcent de rendre leurs services de plus en plus disponibles dans les endroits les plus stratégiques. En 1991, le Québec comptait près de 1500 guichets automatiques; en 1992, les caisses Desjardins en comptaient 1430 à elles seules (Prince, 1993). Les goûts et les préférences des clients changent également d'une manière imprévisible. Ils ne se servent plus de la banque uniquement pour effectuer des dépôts ou des retraits d'argent, mais comme un intermédiaire dans la plupart de leurs transactions financières (paiement direct, assurance, etc.). En 1982, les banques des États-Unis avaient vendu pour près de 1,1 milliard de dollars de certificats de placement garanti contre 296 milliards de dollars de fonds de placement. En 1993, la tendance était inversée : les banques ont vendu pour 1390 milliards de certificats de placement et 1444 milliards de fonds de placement. Que ce soit aux États-Unis ou au Canada, la vente des fonds de placement semble être en croissance (Froment, 1993). Finalement, l'absorption de certains secteurs d'activité par les institutions bancaires (les sociétés de fiducie au Canada, par exemple) ou leur décloisonnement sont en train de transformer complètement l'industrie bancaire.

En raison de ces changements profonds et multiples, les institutions bancaires font face à de plus en plus d'incertitude concernant leur environnement. Aujourd'hui, le but des banques canadiennes, bien qu'elles soient soumises à considérablement moins de concurrence que leurs partenaires des États-Unis, est d'arriver à satisfaire tous les besoins financiers (gestion de portefeuille, intégration des comptes, planification, etc.) de tous leurs clients quel que soit leur âge (Prince, 1993). Dans un tel climat d'incertitude, les banques éprouvent le besoin de mieux s'informer sur leurs clients, sur la concurrence et sur 
le reste de leur environnement afin justement de mieux gérer cette incertitude. Le but de notre étude est d'explorer la manière dont les hauts responsables dans l'industrie bancaire ont réagi à l'incertitude des années 1980-1990 à travers l'utilisation et l'adoption croissante des technologies de l'information.

Le choix du secteur bancaire comme champ d'étude a été guidé par le fait que les banques sont perçues comme des institutions de plus en plus dépendantes de l'information (information intensive) et de la rapidité de sa gestion. En outre, le nombre croissant d'opérations et de transactions bancaires fait que ces entreprises sont également de plus en plus dépendantes des technologies de l'information modernes. Ces considérations font de l'industrie bancaire un secteur de choix pour l'étude des relations entre des construits informationnels (besoins en informations et disponibilité de ces informations), de compétence technologique (degré de complexité technologique et compétence du personnel informatique) et la performance financière des institutions. Notre étude s'inscrit dans un stade exploratoire pour mieux comprendre les relations qui existent entre ces concepts dans un contexte de petites et moyennes entreprises. Nous nous sommes limité aux banques établies aux États-Unis parce qu'elles fournissaient un échantillon beaucoup plus large que celles du Canada et parce que l'incertitude y est beaucoup plus accentuée étant donné leur nombre et la forte concurrence qui en résulte.

\section{La théorie}

L'objectif des technologies de l'information, et plus particulièrement des systèmes d'information, est soit d'augmenter la productivité des cols blancs dans l'accomplissement de leurs tâches les plus routinières, soit d'aider à mettre à leur disposition l'information nécessaire afin qu'ils puissent prendre des décisions plus informées (Bruns et McFarlan, 1987).

Voilà maintenant plus de cinquante ans que l'ordinateur a été inventé et près de quarante ans que les entreprises commerciales l'utilisent (Whistler, 1970). L'hypothèse que l'ordinateur a en effet augmenté la productivité des cols blancs demeure un sujet de controverse (Brynjolfsson, 1993). La deuxième hypothèse (plus de technologie de l'information implique plus d'information) a commencé a être débattue par des chercheurs tels que Galbraith (1977), O'Reilly (1980) et Tushman et Nadler (1978). La conclusion intuitive qui se dégage de ces discussions conceptuelles est que les technologies de l'information doivent être utilisées dans le but de faciliter le stockage et la gestion de l'information, la quantité d'informations à gérer devant être fixée en fonction des besoins en informations des membres de l'entreprise. Ainsi, l'idée de congruence qui vit le jour a été proposée par Galbraith pour déterminer, du 
moins théoriquement, les bornes inférieures et supérieures de la quantité d'informations à gérer. La réalisation des contraintes imposées par le modèle de Galbraith satisfait deux conditions : la première (la borne inférieure) est la condition nécessaire, la seconde (la borne supérieure) est la condition suffisante. La borne inférieure de la quantité d'informations à gérer, donc à rendre disponible, est déterminée par le degré d'incertitude perçue par les gestionnaires (figure 1). Si l'incertitude perçue est d'un degré tel qu'une certaine quantité d'informations devient requise, la quantité d'informations à rendre disponible doit être au moins égale à cette quantité.

\section{Figure 1}

\section{Les relations entre les différents concepts de la théorie} sur l'organisation en tant qu'entité de traitement de l'information

\begin{tabular}{|c|c|c|c|c|c|c|}
\hline & & & Capacité inf & rmati & orga & nisationnelle \\
\hline & & & $\begin{array}{c}\text { Mémoire } \\
\text { organisationnelle }\end{array}$ & orga & nelle & $\begin{array}{c}\text { Technologies } \\
\text { de l'information }\end{array}$ \\
\hline & & & Faible & & & Élevée \\
\hline & & & $\downarrow$ & & & $\downarrow$ \\
\hline & & & Dispc & libilit & matio & anelle \\
\hline & & & Faible & & & Élevée \\
\hline $\begin{array}{l}\qquad \text { Faible } \\
\text { Incertitude } \\
\text { environnementale }\end{array}$ & $\rightarrow$ & $\begin{array}{l}\text { Faibles } \\
\text { Besoins } \\
\text { informationnels }\end{array}$ & (Pefromane & & $\begin{array}{r}\text { In } \\
(\mathrm{Per}\end{array}$ & $\begin{array}{l}\text { congruence } \\
\text { formance } \downarrow \text { ) }\end{array}$ \\
\hline $\begin{array}{l}\text { perçue } \\
\text { Élevée }\end{array}$ & $\rightarrow$ & Élevés & $\begin{array}{l}\text { Incongruer } \\
\text { (Performanc }\end{array}$ & & & $\begin{array}{l}\text { engruence } \\
\text { formance }(1)\end{array}$ \\
\hline
\end{tabular}

La définition de la borne supérieure de la quantité d'informations à rendre disponible est guidée par des considérations d'efficacité et d'efficience. En effet, si la quantité d'informations à rendre disponible satisfait la condition nécessaire, elle peut atteindre des sommets qui résulteraient en une surabondance d'informations (O'Reilly, 1980). Étant donné que la disponibilité de l'information est assurée par divers mécanismes organisationnels tels que la structure (Duncan, 1972 ; Galbraith, 1977), la mémoire organisationnelle 
(Walsh et Ungson, 1991), les technologies de l'information (Bruns et McFarlan, 1987), etc. (figure 1), cet état de fait se traduirait par des investissements considérables qui, bien qu'ils augmentent les avoirs de l'entreprise, pourraient se faire au détriment de sa performance financière.

Si la quantité d'informations disponibles au sein de l'organisation est inférieure à celle requise, une situation suboptimale s'ensuit parce que ses membres doivent se charger eux-mêmes de la recherche d'informations, ce qui a pour effet d'augmenter le temps nécessaire à l'exécution d'une tâche, entraînant une diminution de leur productivité. En bout de ligne, et selon le degré de gravité du problème dans toute l'entreprise, cela pourrait avoir des répercussions sur la performance financière.

L'augmentation de la capacité informationnelle d'une organisation peut se faire par le biais d'investissements dans la ré-ingénierie des processus de travail (Hammer et Champy, 1993), dans la décentralisation et la restructuration des équipes de travail (Byrne, 1993) et dans les technologies de l'information (Gleckman et al., 1993). Si la quantité d'informations disponibles qui en résulte dépasse celle qui est nécessaire, nous nous trouvons dans une situation comme celle décrite par Dearden (1972) où les gestionnaires sont littéralement submergés de rapports et d'états informatiques qui ne sont jamais consultés. L'effet net est double : d'un côté, cela a nécessité des investissements qui auraient pu être faits ailleurs dans l'entreprise et, d'un autre, les gestionnaires répugnent à utiliser un tel volume d'information. Le gestionnaire doit procéder à une recherche de l'information dont il a besoin dans cette avalanche de rapports, ce qu'il aurait probablement fait autrement de toutes façons.

La situation idéale, théoriquement décrite en fonction du concept de congruence par Galbraith, est celle où la quantité d'informations disponibles est égale à la quantité d'informations requises. L'hypothèse de Galbraith (1977), de Tushman et Nadler (1978), de Smith et al. (1991) et d'autres est que la performance d'un système informationnellement « incongruent » est toujours inférieure à celle d'un système informationnellement « congruent ». Dans la figure 1, les zones ombrées indiquent les situations de congruence informationnelle qui, d'après la théorie de Galbraith (1977), correspondent à une performance plus élevée que dans les situations d'incongruence informationnelle.

C'est sur la base de cette théorie et du modèle conceptuel de la figure 1 que nous avons entrepris de recueillir des données pour vérifier empiriquement les relations incertitude environnementale perçue / besoins informationnels, technologies de l'information / disponibilité informationnelle et congruence / performance organisationnelle. Le modèle de recherche que nous avons 
utilisé est basé sur le modèle conceptuel mais ne le décrit pas entièrement. Ainsi, nous nous sommes limité à un seul aspect de la capacité informationnelle organisationnelle : les technologies de l'information.

\section{La méthodologie}

\subsection{L'échantillon}

Nous avons entrepris notre enquête aux États-Unis entre le mois de novembre 1991 et le mois de janvier 1992. En 1991, il y avait 63321 unités bancaires aux États-Unis (Rand McNally Bankers Directory, 1991). Parce que nous avons inclus une mesure de l'incertitude environnementale, nous avons sélectionné les unités bancaires (agences, succursales, etc.) qui étaient classifiées comme des banques commerciales nationales (SIC : 6021) ou des banques commerciales d'État (SIC : 6022) ${ }^{1}$. Il importait que les banques ainsi choisies ne soient orientées que sur un seul type d'activité (un seul code SIC) pour contrôler l'effet de l'environnement objectif que nous n'avons pas mesuré et qui a été identifié comme une source de problèmes considérables dans toute tentative de mesure de l'incertitude environnementale (voir le débat entre Lawrence et Lorsch, 1967 ; Tosi et al., 1973a; Lawrence et Lorsch, 1973; Tosi et al., 1973 b; et la discussion de Downey et Ireland, 1979 à ce sujet). En n'ayant qu'un seul type d'activité, les banques de notre échantillon n'ont qu'un seul environnement objectif ; ce qui variera sera l'incertitude environnementale perçue.

Notre choix d'une seule industrie a également été guidé par la nécessité de mesurer un seul type d'incertitude environnementale. Comme chaque industrie a ses propres caractéristiques et ses propres sources d'incertitude (Hambrick, 1981), l'échantillon devait contenir une certaine variance pour la mesure de l'incertitude environnementale perçue mais demeurer comparable. Pour ce faire, et en sélectionnant uniquement le secteur bancaire, nous avons contrôlé la variance interindustrie pour ne garder que la variance intraindustrie.

Parmi ces 63321 unités bancaires, seules 986 répondaient aux trois critères de sélection suivants : 1) compter 300 employés ou moins, 2) avoir un seul code SIC et 3 ) être une banque commerciale, c'est-à-dire avoir le code SIC égal à 6021 ou 6022 . Nous avons utilisé 100 unités pour tester notre ques-

1. Aux États-Unis, le SIC (Standard Industrial Code) indique le secteur d'affaires principal d'une entreprise commerciale. La classification SIC a été mise en place par le département du Commerce américain. Ainsi un code détermine l'activité qui comprend plus de $10 \%$ des revenus d'une entreprise. 
tionnaire et valider nos mesures. Parmi les 886 unités restantes, 288 ont participé à l'enquête après un envoi et un rappel. Ainsi, près d'un tiers $(32,50 \%)$ de l'échantillon visé a participé à l'étude.

De ces 288 banques, 190 comptaient 300 employés ou moins et ont été considérées pour les analyses qui suivent. Notre intérêt pour les banques de petite et moyenne tailles se justifie par les contraintes financières (Cohn et Lindberg, 1972) et les insuffisances en matière de gestion de l'information (DeLone, 1981) qui caractérisent les PME en général. Le critère du nombre d'employés que nous avons utilisé est basé sur Sheshunoff qui considère qu'une banque de moins de 301 employés constitue l'équivalent d'une PME pour le secteur bancaire. Les caractéristiques de l'échantillon de l'étude sont telles que décrites dans le tableau 1.

\section{TABLEAU 1}

Caractéristiques des banques de l'échantillon

$(n=190)$

\begin{tabular}{lccccc}
\hline Variable & Minimum & Maximum & Moyenne & Médiane & Écart type \\
\hline Nombre d'employés & 24 & 300 & 133 & 119 & 70.20 \\
Rendement de l'actif' & $-1.99 \%$ & $2,76 \%$ & $0,93 \%$ & $1,02 \%$ & $0,58 \%$ \\
Rendement de l'avoir & & & & & \\
des actionnaires $^{1}$ & $-30,77 \%$ & $32,48 \%$ & $11,56 \%$ & $12,81 \%$ & $7,63 \%$ \\
Revenu net moyen $^{1,2}$ & -6287 & 380000 & 4274 & 1759 & 27873 \\
Avoirs moyens $^{1,2}$ & 26000 & 1000000 & 253000 & 218000 & 156000 \\
\hline
\end{tabular}

1. Moyenne sur les quatre ans précédant l'enquête (1988-1991).

2. En milliers de dollars américains.

\subsection{Le questionnaire et les mesures}

Le questionnaire de cinq pages contenait autant de sections que de variables mesurées : l'incertitude environnementale perçue, la quantité d'informations dont le répondant (un président ou un directeur général) avait besoin dans ses tâches quotidiennes, la quantité de ces informations qui était disponible, la sophistication technologique, la compétence du personnel informatique perçue et différents indicateurs subjectifs de performance. Nous avons également pu obtenir les indicateurs factuels de performance financière de ces banques grâce à l'achat de la banque de données commercialisées par Sheshunoff Information Services, Inc. qui se spécialise dans le secteur bancaire et qui compile ces indicateurs à partir des bandes magnétiques de la Federal Deposit Insurance Commission (FDIC). Nous avons pu corroborer la valeur des indicateurs Sheshunoff d'un sous-échantillon aléatoire avec des résultats publiés dans Business Week, Rand McNally Directory, Value Line Survey et Forbes. 
Nous avons adapté la mesure de l'incertitude environnementale perçue de Gordon et Narayanan (1984) qui est composée de neuf questions (tableau 2). La quantité d'informations était mesurée par des questions relatives à neuf domaines de l'environnement externe inspirés des travaux de Duncan (1972) et Ghymn et King (1976). Les répondants devaient indiquer sur une échelle de type Likert allant de 1 -aucun besoin pour les besoins informationnels et non disponible pour la disponibilité informationnelle - à 5 - grand besoin et très disponible respectivement pour les besoins et la disponibilité informationnels - relativement à chacun des neuf domaines (tableau 2).

La notion de congruence entre deux variables peut être mesurée de diverses manières (Drazin et Van de Ven, 1985 ; Venkatraman, 1989). Venkatraman en répertorie trois : l'analyse des résidus obtenus à partir de la régression d'une des deux variables sur l'autre, l'analyse des variances et l'analyse des scores de déviation calculés à partir de la valeur absolue de la différence entre deux variables. La première approche n'est pas décisive quant au choix de la variable à faire régresser et au choix de la valeur à choisir pour déterminer les différents degrés de congruence. L'analyse des variances est généralement plus appropriée pour tester l'existence d'interaction entre deux variables ou pour le cas où au moins une des deux variables entre lesquelles le degré de congruence est calculé est mesurée sur une échelle qui n'est pas de type intervalle. L'analyse des scores de déviation, par contre, présente l'avantage d'être simple et applicable aux modèle et variables de cette étude (voir aussi la comparaison de Drazin et Van de Ven, 1985, à la page 522). Nous avons, par conséquent, formé notre variable de la congruence en 1) calculant la valeur absolue de la différence arithmétique entre besoin et disponibilité au niveau de chacune des neuf questions et en 2) calculant la moyenne arithmétique de ces différences. Nous avons enfin transformé la valeur de la mesure que nous avons obtenue pour qu'elle reflète la valeur de la congruence conceptuelle ${ }^{2}$.

2. Si la valeur de la congruence mesurée est égale à zéro, nous sommes en présence d'un cas de congruence conceptuelle élevée ; si la valeur de la congruence mesurée est égale à cinq, nous sommes en présence d'une faible congruence conceptuelle. Pour contourner cette difficulté, nous avons déduit la valeur mesurée de la valeur maximale possible (5) afin d'obtenir une valeur qui corresponde à la congruence conceptuelle. Nous avons utilisé le résultat de cette opération dans toutes nos analyses. 


\section{TABleau 2}

\section{Les variables mesurées, leurs indicateurs et leurs caractéristiques statistiques}

\section{Incertitude environnementale perçue}

1. Intensité de la concurrence pour la compétence humaine

2. Intensité de la concurrence basée sur les taux tarifaires

$\min . \quad 2,22$ des produits et services

$\max .: \quad 4,67$

3. Nombre de nouveaux produits et services qui ont été introduits dans l'industrie dans les cinq dernières années

moy. : $\quad 3,57$

$\sigma: \quad 0,44$

$\alpha: \quad 0,65$

4. Stabilité de l'environnement économique

5. Stabilité de l'environnement technologique

6. Activités des concurrents

7. Goûts et préférences des clients

8. Contraintes réglementaires

9. Innovations dans les secteurs bancaires

\section{Quantité d'informations (besoins et disponibilités)}

1. Clients de la banque

2. Disponibilité du personnel compétent sur le marché de l'emploi

3. Situation concurrentielle de la banque

4. Concurrents de la banque

5. Gouvernement et la réglementation

6. Critères de rémunération des employés ailleurs que dans votre banque

7. Affaires internationales

8. Exigences technologiques de l'industrie bancaire

9. Forces et faiblesses de vos produits et services dans le marché

\section{Besoins}

min. : $\quad 1,78$

$\max .: \quad 5,00$

moy. : $\quad 3,68$

$\sigma: \quad 0,50$

$\alpha: \quad 0,75$

Disponibilités

min. : $\quad 2,22$

$\max .: \quad 4,78$

moy. : $\quad 3,56$

$\sigma: \quad 0,49$

$\alpha: \quad 0,74$

\section{Congruence informationnelle}

Moyenne arithmétique de la valeur absolue des différences entre besoins et disponibilités avec la différence calculée au niveau de chaque question et le résultat inversé (différence arithmétique élevée $=$ faible congruence)

min. : $\quad 0,00$

$\max .: \quad 3,00$

moy. : $\quad 0,87$

$\sigma: \quad 0,39$

$\alpha: \quad 0,71$ 
TABleau 2 (SUITE)

Les variables mesurées, leurs indicateurs et leurs caractéristiques statistiques

\section{Sophistication technologique}

1. Calendriers électroniques et supports de planification

2. Appareils de traitement électronique de l'image

$\min .: \quad 1,00$ (digitaliseurs, etc.)

$\max .: 5,50$

moy. : $\quad 2,64$

3. Systèmes experts pour les décisions sur les prêts bancaires

$\sigma: \quad 0,97$

4. Traitement en temps réel des comptes recevables et à payer

$\alpha: \quad 0,76$

5. Transactions bancaires par téléphone

6. Traitement des demandes de prêts

7. Messagerie électronique

8. Messagerie vocale

9. Réseaux de données (locaux, distribués, etc.)

10. Guichets automatiques

\section{Compétence informatique perçue}

1. Compétence des analystes en informatique en tout ce qui concerne les applications des utilisateurs

$\min .: \quad 1,63$

$\max .: \quad 5,00$

2. Qualité des analystes en informatique

moy.: $\quad 3,23$

3. Compétence des analystes en informatique

$\sigma: \quad 0,62$

4. Compétence technique du personnel du département informatique $\alpha$ : $\quad 0,84$

5. Rapidité avec laquelle le personnel informatique satisfait les requêtes et demandes des utilisateurs

6. Degré de collaboration du personnel informatique en ce qui concerne les demandes de mise à jour et de maintenance des applications existantes

7. Degré de collaboration du personnel informatique en ce qui concerne les demandes de conception et de mise en place de nouvelles applications

8. Excellence de la formation et des services dispensés aux utilisateurs par le personnel informatique

\begin{tabular}{lll}
\hline \multicolumn{2}{c}{ Profit perçu } & \\
\hline 1. Rendement de l'actif & min. : & 1,00 \\
2. Rendement de l'avoir des actionnaires & max. : & 5,00 \\
3. Revenu net & moy. : & 3,67 \\
& $\sigma:$ & 1,11 \\
& $\alpha:$ & 0,95 \\
\hline
\end{tabular}


TABleau 2 (SUITE)

\section{Les variables mesurées, leurs indicateurs et leurs caractéristiques statistiques}

\section{Taux de croissance perçu}

1. Taux de croissance (sur 4 ans) des dépôts et des prêts relativement à la concurrence

min. : $\quad 1,00$

$\max .: \quad 5,00$

2. Taux de croissance (sur 4 ans) du revenu brut relativement à la concurrence

moy. : $\quad 3,45$

$\sigma: \quad 0,89$

$\alpha: \quad 0,80$

\section{Performance financière globale}

$=1$. Performance financière globale par rapport à la concurrence

\begin{tabular}{lr} 
min. : & 1,00 \\
max. : & 5,00 \\
moy. : & 3,75 \\
$\sigma:$ & 1,10 \\
$\alpha:$ & s.o. \\
\hline
\end{tabular}

La sophistication technologique d'une organisation a été définie comme la présence d'une caractéristique technologique particulière par Srinivasan (1985). Floyd et Wooldridge (1990) ont étendu cette mesure à une échelle de type Likert qui indique le degré d'implantation d'une technologie particulière lorsqu'elle est présente dans une organisation. Nous avons utilisé la liste des technologies de l'information de Floyd et Wooldridge et nous l'avons complétée avec d'autres technologies plus spécifiques au secteur bancaire après avoir consulté des publications spécialisées dans ce secteur. La liste initiale utilisée dans le prétest comprenait 27 technologies. Une analyse psychométrique nous a permis de n'en garder que 10 (tableau 2). L'échelle utilisée était la suivante :

1) pas opérationnelle et non considérée à long terme ;

2) en considération mais nous n'avons pas encore de schéma d'implantation pour cette technologie ;

3) en pleine planification pour implantation à court terme ;

4) opérationnelle en tant que projet pilote ;

5) en cours d'implantation ;

6) opérationnelle sur une grande échelle.

La mesure de la compétence du personnel informatique perçue a été mesurée sur une échelle du type Likert allant de 1 à 5 à l'aide de huit questions portant sur la compétence, la qualité et l'excellence des services du personnel informatique. 
Nous avons formé nos mesures de profit et de croissance subjectives en calculant la moyenne des critères correspondants donnés dans le tableau 2. Pour les mesures de performance factuelle, les indicateurs suivants étaient disponibles pour chacune des années qui se sont écoulées entre 1988 et 1991 dans la banque de données de Sheshunoff : 1) le rendement de l'actif, 2) le rendement de l'avoir des actionnaires, 3) le revenu net, 4) le taux de croissance (sur 4 ans) des dépôts et des prêts et 5 ) le taux de croissance (sur 4 ans) du revenu brut. Compte tenu du fait que ces indicateurs avaient des unités de mesure différentes (dollars et pourcentages), nous les avons standardisés pour obtenir des scores $z$. Nous avons ensuite formé un indicateur de profit financier factuel et un indicateur de croissance factuelle en procédant de la même manière qu'avec les mesures de performance subjectives.

Tous les coefficients de fidélité interne des mesures (a de Cronbach) sont supérieurs à 0,70 à l'exception de la mesure de l'incertitude environnementale. De plus, aucun des coefficients de précision a (Cortina, 1993) n'était significativement différent de zéro, ce qui signifie que toutes les mesures sont unidimensionnelles.

\section{Les résultats}

Les premiers résultats de l'analyse des corrélations entre les variables du modèle sont présentés au tableau 3 .

Comme nous pouvons le constater, les variables qui sont significativement et positivement corrélées sont 1) l'incertitude environnementale et les besoins informationnels, 2) la sophistication technologique et la disponibilité informationnelle et 3) la compétence informatique perçue et la disponibilité informationnelle. Ces résultats confirment nos attentes basées sur le modèle de la figure 1 .

Nous constatons également une corrélation positive et significative entre la mesure subjective de la croissance et 1) la sophistication technologique et 2) les besoins informationnels ainsi qu'entre la compétence informatique perçue et l'indicateur factuel de croissance.

Nous sommes cependant étonné du manque d'association entre la congruence et les différents indicateurs de performance. La congruence ne semble être associée qu'à la sophistication et à la compétence informatique perçue.

Finalement, nous relevons les fortes corrélations entre les différents indicateurs de performance, qu'ils soient subjectifs ou factuels $(r=0,92$, $\mathrm{p}<0,0001$ ). Cela semble indiquer que les banquiers participants avaient une 
Tableau 3

Corrélations entre les différentes variables du modèle $(174<n<190)$

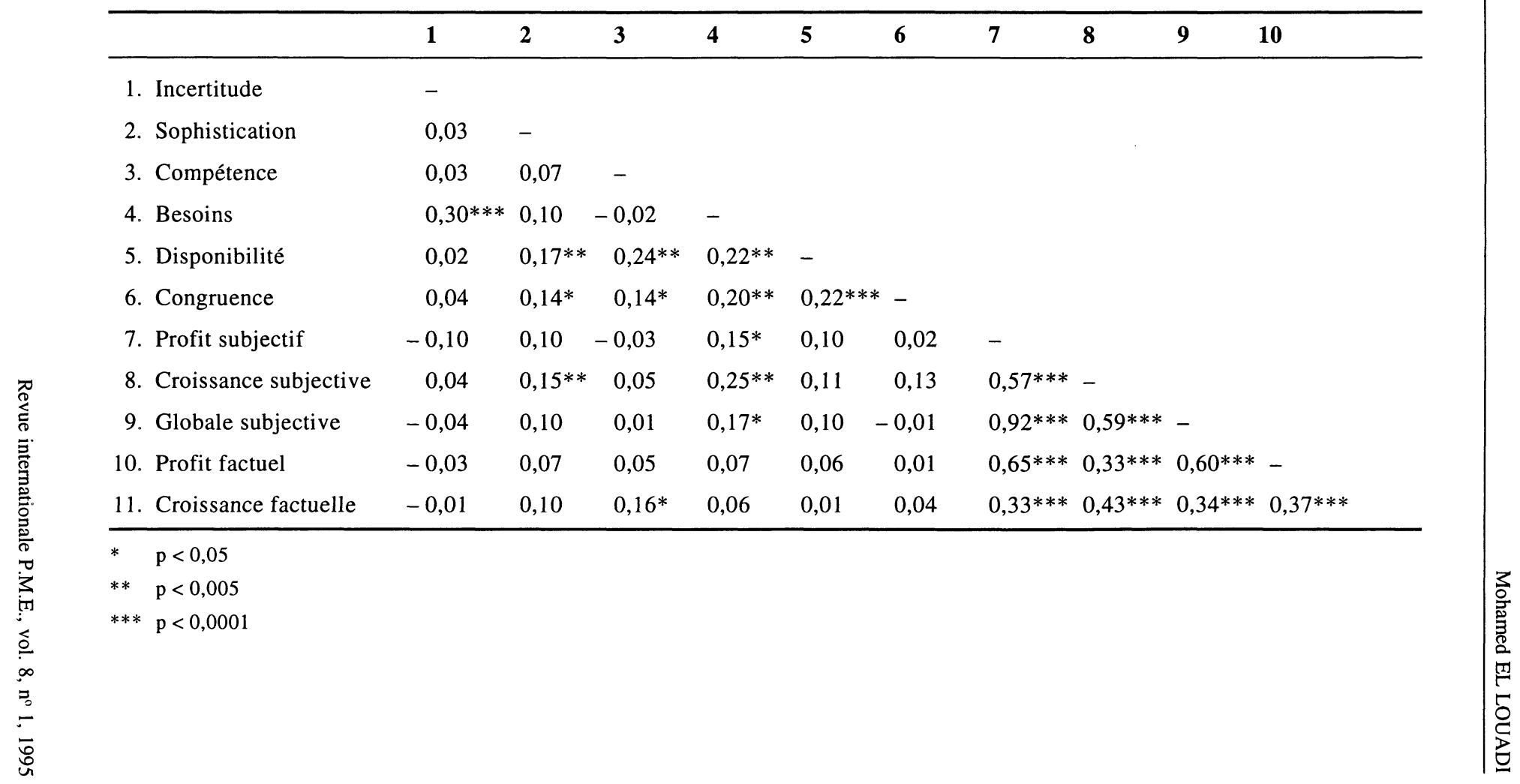


idée passablement précise de la performance réelle de leurs banques. Nous noterons au passage qu'entre tous ces coefficients, celui reliant l'indicateur de performance globale subjective à celui du profit est le plus élevé. Cela suggère que dans l'esprit des répondants, performance globale signifie d'abord profit.

Compte tenu de l'absence de corrélation entre la congruence et la performance financière des banques de notre échantillon, et vu que la théorie prévoyait que le degré de congruence variait entre les banques sur la base de la performance, nous avons entrepris de diviser l'échantillon en fonction de tous les critères de performance disponibles. Comme il n'était pas possible d'utiliser simultanément les médianes ou les moyennes des cinq indicateurs de performance comme critère de division, nous avons eu recours à une analyse typologique.

\section{L'analyse typologique}

L'analyse typologique a l'avantage de séparer un échantillon en plusieurs groupes considérant non seulement la moyenne mais aussi la variance des variables critères. En optant pour l'analyse typologique hiérarchique avec distances euclidiennes, nous avons cherché à obtenir deux groupes tels que la variance à l'intérieur d'un groupe soit minimale et que la variance entre les groupes soit maximale. Cette méthode impose des contraintes considérables sur la formation des groupes (Churchill, 1979). Afin de vérifier la validité post-hoc de la typologie, nous avons utilisé le test de Scheffé qui est considéré comme l'un des tests de comparaison multivariés les plus conservateurs (Huck et al., 1974). Il présente également l'avantage de s'appliquer à des groupes de tailles différentes et d'être relativement insensible aux violations des hypothèses de normalité et d'homogénéité des variances (Hays, 1963). Les résultats de ces tests montrent que les deux groupes sont différents au niveau de signification $\mathrm{p}<0,0001$.

Les résultats du tableau 4 montrent la validation post-hoc du résultat de l'analyse typologique (dichotomie) sur les variables critères que sont les cinq indicateurs de performance. Il indique aussi que les entreprises du premier groupe sont en moyenne significativement plus performantes que celles du deuxième groupe. Il révèle finalement que les entreprises des deux groupes ont des profils technologiques et informationnels similaires et qu'elles ne se différencient pas non plus dans la manière de percevoir leur environnement.

Compte tenu de ces résultats, nous avons entrepris d'explorer les différences sur le plan des associations entre les variables du modèle (tableau 5). C'est au tableau 5 que les différences sont le mieux révélées. Comme nous pouvons le constater, les différences ne se situent pas dans les moyennes des 
variables mesurées mais dans les relations qui existent entre elles. Nous explorons ces relations dans les prochaines sections en comparant les résultats du tableau 5 à ceux du tableau 3.

\subsection{L'incertitude environnementale perçue}

La première comparaison que nous avons effectuée montre que c'est en fait les entreprises du premier groupe qui ont amené la corrélation de 0,30 entre l'incertitude et les besoins informationnels du tableau 3. Si cette relation persiste dans le cas du deuxième groupe ( $r=0,23$; tableau 5$)$, elle n'est pas significative. Si le coefficient de corrélation est indépendant de la taille de l'échantillon (Cohen et Cohen, 1983) ${ }^{3}$ - pourvu que l'échantillon contienne plus de deux éléments -, cela n'est pas le cas du degré de signification qui est directement dépendant de $n$.

\subsection{La sophistication technologique}

Les relations entre la sophistication technologique, d'une part, et la disponibilité et la congruence informationnelles, de l'autre, étaient positives et significatives dans tout l'échantillon (tableau 3) mais ne le sont plus quand celui-ci est subdivisé en deux groupes (tableau 5). La relation entre la sophistication et les besoins informationnels n'est significative ni dans le tableau 3 ni dans le cas du premier groupe du tableau 5 , mais elle l'est dans le cas du deuxième groupe. En fait, il semblerait que c'est le très faible coefficient du premier groupe $(r=0,02$; tableau 5$)$ qui ait fait que cette relation ne soit plus détectable dans l'ensemble de l'échantillon. Cela ne semble pas avoir été le cas de la relation entre la sophistication technologique et le taux de croissance qui est significative dans le cas de tout l'échantillon $(\mathrm{r}=0,15 ; \mathrm{p}<0,005$, tableau 3 ) et dans celui du deuxième groupe ( $\mathrm{r}=0,30 ; \mathrm{p}<0,05$, tableau 5).

\subsection{La compétence informatique perçue}

La relation entre la compétence informatique perçue et la disponibilité informationnelle est l'une des rares relations qui soient restées positives et significatives après la subdivision de l'échantillon. La relation entre la compétence et la congruence, qui était significative dans le tableau 3 $(\mathrm{r}=0,14 ; \mathrm{p}<0,05)$, ne l'est plus que dans le cas du deuxième groupe du tableau $5(\mathrm{r}=0,26 ; \mathrm{p}<0,05)$. Le phénomène inverse a eu lieu pour la relation

3. Comme nous pouvons le constater dans la formule suivante, $n$ (la taille de l'échantillon) est complètement absent dans le calcul de $r$. 
TABLEAU 4

Résultats du test de Scheffé sur les moyennes des variables

(degrés de liberté $=\mathbf{1 , 1 8 8}$ )

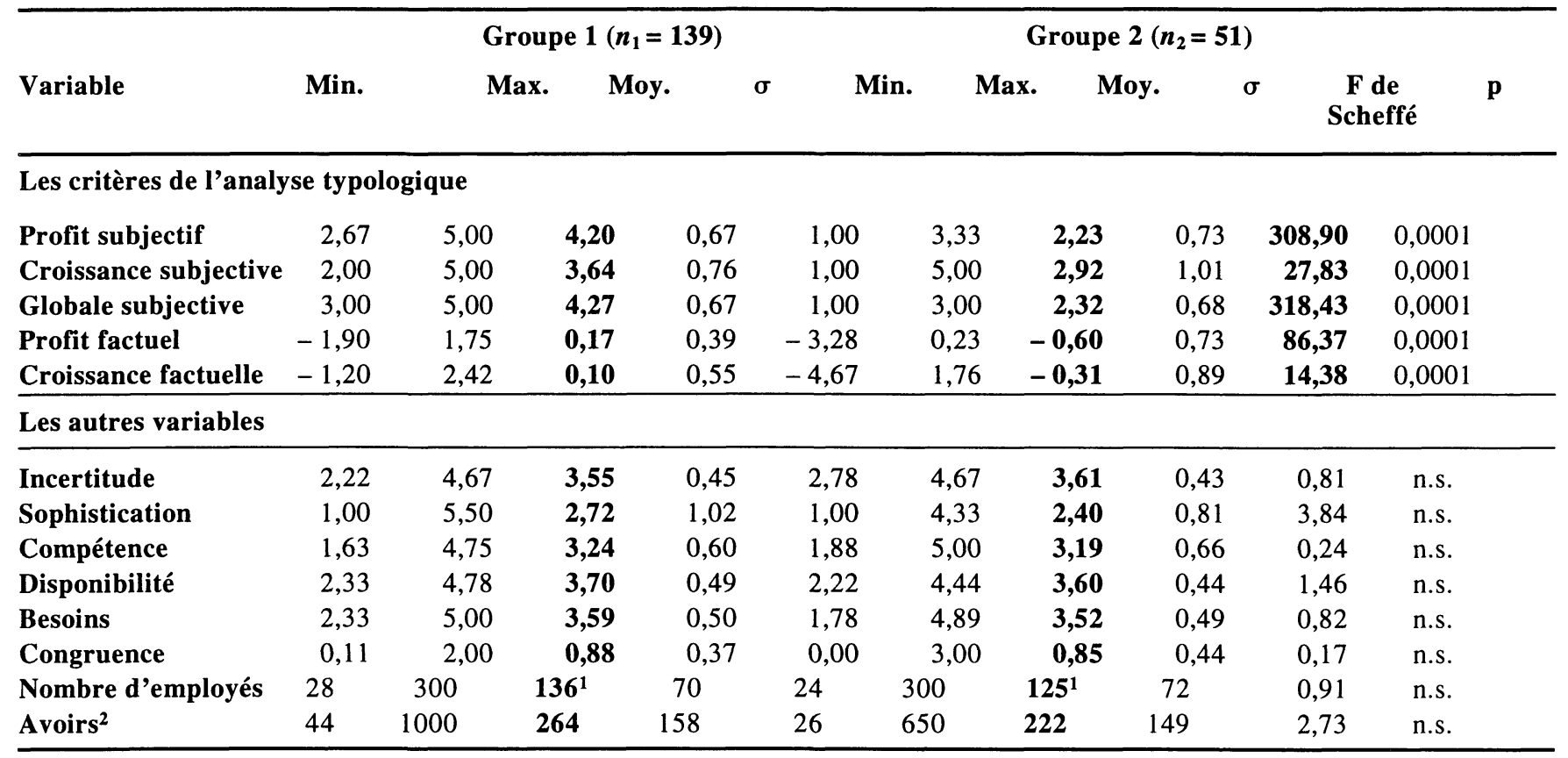

1. Médianes.

2. En milliards de dollars américains.

Note : Les banques du groupe 1 sont considérablement plus performantes que celles du groupe 2 . 
TABLEAU 5

Corrélations entre les différentes variables du modèle après la subdivision

\begin{tabular}{|c|c|c|c|c|c|c|c|c|c|c|}
\hline & 1 & 2 & 3 & 4 & 5 & 6 & 7 & 8 & 9 & 10 \\
\hline \multicolumn{11}{|c|}{ groupe $1\left(130<n_{1}<138\right)$} \\
\hline 1. Incertitude & - & & & & & & & & & \\
\hline 2. Sophistication & 0,01 & - & & & & & & & & \\
\hline 3. Compétence & 0,10 & 0,06 & _- & & & & & & & \\
\hline 4. Besoins & $0,33 * * *$ & $* 0,02$ & $-0,06$ & - & & & & & & \\
\hline 5. Disponibilité & 0,05 & 0,14 & $0,19 *$ & $0,19 *$ & - & & & & & \\
\hline 6. Congruence & 0,02 & 0,14 & 0,08 & 0,04 & $0,28 * *$ & _- & & & & \\
\hline 7. Profit $(s)^{1}$ & $-0,02$ & $-0,01$ & $-0,11$ & 0,14 & 0,13 & 0,03 & _- & & & \\
\hline 8. Croissance (s) & 0,14 & 0,04 & 0,02 & $0,20 *$ & 0,09 & 0,07 & $0,43 * * *$ & - & & \\
\hline 9. Globale (s) & 0,07 & $-0,05$ & $-0,10$ & 0,15 & 0,08 & 0,12 & $0,75 * * *$ & $0,50 * * *$ & - & \\
\hline 10. Profit (f) & $-0,07$ & 0,01 & 0,04 & 0,07 & $-0,01$ & 0,03 & $0,44 * * *$ & 0,04 & $0,28 * *$ & - \\
\hline 11. Croissance (f) & 0,10 & $-0,01$ & $0,21 *$ & 0,10 & $-0,07$ & 0,04 & 0,05 & $0,27 * *$ & 0,06 & 0,03 \\
\hline
\end{tabular}

groupe $2\left(47<\mathbf{n}_{2}<51\right)$

\begin{tabular}{|c|c|c|c|c|c|c|c|c|c|}
\hline 1. Incertitude & - & & & & & & & & \\
\hline 2. Sophistication & 0,16 & - & & & & & & & \\
\hline 3. Compétence & $-0,13$ & 0,04 & - & & & & & & \\
\hline 4. Besoins & 0,23 & $0,32 *$ & 0,09 & - & & & & & \\
\hline 5. Disponibilité & 0,02 & 0,28 & $0,39 * *$ & $0,41 * *$ & - & & & & \\
\hline 6. Congruence & 0,11 & 0,18 & $0,26^{*}$ & $0,58 * * *$ & 0,07 & - & & & \\
\hline 7. Profit (s) & $-0,21$ & $-0,05$ & $-0,04$ & 0,09 & 0,10 & $0,30 *$ & - & & \\
\hline 8. Croissance (s) & $-0,08$ & $0,30 *$ & 0,07 & $0,31 *$ & 0,11 & $0,28 *$ & $0,63 * * *$ & - & \\
\hline 9. Globale (s) & $-0,09$ & 0,05 & 0,02 & 0,20 & 0,08 & $0,33 *$ & $0,84 * * *$ & $0,61 * * *$ & - \\
\hline 0. Profit (f) & 0,10 & $-0,05$ & 0,07 & 0,16 & 0,09 & 0,11 & $0,41 * *$ & $0,31 *$ & $0,36 *$ \\
\hline 11. Croissance (f) & $-0,19$ & 0,26 & 0,03 & 0,28 & 0,11 & 0,21 & $0,45 * *$ & $0,50 * * *$ & $0,50 * * * 0,49 * * *$ \\
\hline
\end{tabular}

1. (s) subjectif et (f) factuel.

$* \quad \mathrm{p}<0,05$

$* * \quad \mathrm{p}<0,005$

$* * * \mathrm{p}<0,0001$

Note: Les banques du groupe 1 sont considérablement plus performantes que celles du groupe 2 (tableau 4). 
entre la compétence et le taux de croissance factuel qui n'est demeuré significatif que dans le cas du premier groupe $(r=0,21 ; p<0,05)$.

\subsection{Les besoins informationnels}

À l'instar de la relation entre la compétence et la disponibilité, la relation entre les besoins informationnels et la disponibilité est demeurée significative pour les deux groupes. Cette relation est positive et significative pour l'échantillon pris dans sa totalité $(r=0,22 ; \mathrm{p}<0,005)$, pour les banques du premier groupe $(\mathrm{r}=0,19 ; \mathrm{p}<0,05)$ et pour celles du deuxième groupe $(\mathrm{r}=0,41 ; \mathrm{p}<0,005)$. Les besoins informationnels sont aussi corrélés avec les trois indicateurs subjectifs de la performance dans le tableau 3. Si la relation avec le taux de croissance subjectif reste positive et significative dans tous les cas, celles reliant les besoins informationnels aux profits et aux performances globales subjectives ne sont plus significatives après la subdivision.

$$
\mathrm{r}=\frac{\sum x y}{\sqrt{\sum x^{2} \sum y^{2}}}
$$

\subsection{La congruence informationnelle}

Dans le tableau 3, la congruence informationnelle ne semble être associée à aucun indicateur de performance, ce qui contredit la théorie de Galbraith (1977). Seules des relations avec les indicateurs de performance subjectifs sont devenues significatives après la subdivision. Ces relations sont positives et significatives $(\mathrm{r}=0,30 ; 0,28$ et 0,$33 ; \mathrm{p}<0,05$ respectivement pour le profit, la croissance et la performance globale), et elles ne se sont révélées que dans le cas des banques du deuxième groupe (voir le tableau 5).

Tous ces résultats sont résumés dans le tableau 6 où nous reportons également le degré de signification des différences entre les coefficients de corrélation des deux groupes. La signification de la différence entre deux coefficients de corrélation est donnée par la formule de Cohen et Cohen (1983) qui, à partir du test conservateur $\mathrm{z}$ de Fisher, calcule le degré de signification de ces différences.

Nous avons catégorisé les relations en les classifiant en cinq types selon qu'elles sont significatives dans l'échantillon total et les deux groupes (type 1), seulement dans l'échantillon total (type 2), seulement dans le groupe 2 (type 3), dans l'échantillon total et seulement dans le groupe 1 (type 4) et dans l'échantillon total et seulement dans le groupe 2 (type 5). Nous 
avons de plus numéroté les relations individuellement afin de faciliter la lecture du tableau 6 à partir des interprétations que nous proposons dans la section suivante.

\section{Les interprétations}

Notre première analyse sur un échantillon de 190 banques de 300 employés ou moins ne nous a permis de déceler qu'un certain nombre de relations entreles variables que nous avons mesurées. Nous avons été en outre incapable de vérifier l'association positive entre la congruence informationnelle et lesindicateurs de performance que nous avons utilisés.

Une deuxième analyse basée sur la subdivision de notre échantillon en deux groupes nous a permis de trouver des relations qui n'étaient pas décelables dans notre première analyse. Si aucune différence significative n'a pu être établie parmi les moyennes des variables, en revanche nous avons pu en trouver dans les relations entre ces variables.

Le tableau 6 illustre bien les relations qui sont significatives dans tout l'échantillon et pour les deux groupes (relations de type 1). Ainsi, la compétence informatique perçue serait importante dans la perception qu' ont les banquiers que l'information dont ils ont besoin est disponible. Cette importance est accentuée chez les banques du deuxième groupe (les moins performantes financièrement). L'association entre les besoins en informations et la disponibilité informationnelle est aussi plus significative dans le cas de ce groupe. La question demeurera de savoir si ce sont les besoins qui déterminent la disponibilité ou le contraire. À noter que ce sont les besoins informationnels et non la disponibilité informationnelle qui est associée à la croissance subjective des banques.

Les relations de type 2 sont celles qui ne sont significatives que dans le cas où l'échantillon tout entier est considéré. Nous pourrions croire que ces relations cessent d'être significatives parce que la taille des sous-échantillons est inférieure par définition à celle de tout l'échantillon. Ces relations montrent que la sophistication technologique augmente la perception que l'information dont on a besoin est disponible. L'hypothèse du contraire est à peine plausible. Cela nous permet d'avancer que les investissements en technologies de l'information ont au moins l'effet d'augmenter cette perception. Mais est-ce financièrement productif ? La réponse à cette question ne se trouve pas là où la relation sophistication-disponibilité ( ${ }^{\circ} 4$ dans le tableau 6$)$ a été observée mais dans les banques du groupe 2, même si la relation sophisticationcongruence (relation $\mathrm{n}^{\circ} 5$ ) a aussi été observée dans tout l'échantillon. 
Tableau 6

Résultats des comparaisons entre les relations après la subdivision $\left(n_{1}=139, n_{2}=51\right)$

\begin{tabular}{|c|c|c|c|c|c|c|c|c|}
\hline \multicolumn{2}{|r|}{ Types de relations } & \multicolumn{3}{|c|}{ Variables de la relation } & \multirow{2}{*}{$\begin{array}{c}\begin{array}{c}\text { Degré de } \\
\text { signification } \\
\text { pour tout } \\
\text { l'échantillon }\end{array} \\
\mathbf{0 , 2 4 * *} \\
\mathbf{0 , 2 2} * * \\
\mathbf{0 , 2 5} * *\end{array}$} & \multirow{2}{*}{$\begin{array}{c}\begin{array}{c}\text { Degré de } \\
\text { signification } \\
\text { pour le } \\
\text { groupe } 1\end{array} \\
0,19 * \\
0,19 * \\
0,20 *\end{array}$} & \multirow{2}{*}{$\begin{array}{c}\begin{array}{c}\text { Degré de } \\
\text { signification } \\
\text { pour le } \\
\text { groupe 2 }\end{array} \\
\mathbf{0 , 3 9 * *} \\
\mathbf{0 , 4 1 * *} \\
\mathbf{0 , 3 1} *\end{array}$} & \multirow{2}{*}{$\begin{array}{c}\begin{array}{c}\text { Signification } \\
\text { de la } \\
\text { différence } \\
(\boldsymbol{p})\end{array} \\
\mathbf{0 , 0 9 7} \\
\mathbf{0 , 0 7 4} \\
\text { n.s }\end{array}$} \\
\hline 1 & $\begin{array}{l}\text { Relations qui sont } \\
\text { positives et significa- } \\
\text { tives dans tous les cas }\end{array}$ & $\begin{array}{l}\mathbf{1} \\
\mathbf{2} \\
\mathbf{3}\end{array}$ & $\begin{array}{l}\text { compétence } \\
\text { besoins } \\
\text { besoins }\end{array}$ & $\begin{array}{l}\text { disponibilité } \\
\text { disponibilité } \\
\text { croissance (s) }\end{array}$ & & & & \\
\hline 3 & $\begin{array}{l}\text { Relations qui ne sont } \\
\text { significatives que dans } \\
\text { le deuxième groupe }\end{array}$ & $\begin{array}{r}8 \\
9 \\
10 \\
11\end{array}$ & $\begin{array}{l}\text { sophistication } \\
\text { congruence } \\
\text { congruence } \\
\text { congruence }\end{array}$ & $\begin{array}{l}\text { besoins } \\
\text { profit }(\mathrm{s}) \\
\text { croissance }(\mathrm{s}) \\
\text { performance } \\
\text { globale }(\mathrm{s})\end{array}$ & $\begin{array}{l}0,10 \\
0,13 \\
0,01 \\
0,01\end{array}$ & $\begin{array}{l}0,02 \\
0,03 \\
0,07 \\
0,12\end{array}$ & $\begin{array}{l}\mathbf{0 , 3 2 *} \\
\mathbf{0 , 3 0 *} \\
\mathbf{0 , 2 8} * \\
\mathbf{0 , 3 3} *\end{array}$ & $\begin{array}{l}0,036 \\
0,049 \\
0,097 \\
0,097\end{array}$ \\
\hline 4 & $\begin{array}{l}\text { Relations qui ne sont } \\
\text { pas significatives } \\
\text { uniquement dans le } \\
\text { groupe } 2\end{array}$ & $\begin{array}{l}12 \\
13\end{array}$ & $\begin{array}{l}\text { incertitude } \\
\text { compétence }\end{array}$ & $\begin{array}{l}\text { besoins } \\
\text { croissance (f) }\end{array}$ & $\begin{array}{l}\mathbf{0 , 3 0 * * *} \\
\mathbf{0 , 1 6 *}\end{array}$ & $\begin{array}{l}\mathbf{0 , 3 3} * * * \\
\mathbf{0 , 2 1} *\end{array}$ & $\begin{array}{l}0,23 \\
0,03\end{array}$ & $\begin{array}{l}\text { n.s. } \\
\text { n.s. }\end{array}$ \\
\hline 5 & $\begin{array}{l}\text { Relations qui ne sont } \\
\text { pas significatives uni- } \\
\text { ment dans le groupe } 1\end{array}$ & $\begin{array}{l}14 \\
15\end{array}$ & $\begin{array}{l}\text { compétence } \\
\text { sophistication }\end{array}$ & $\begin{array}{l}\text { congruence } \\
\text { croissance (s) }\end{array}$ & $\begin{array}{l}0,14 * \\
0,15 * *\end{array}$ & $\begin{array}{l}0,08 \\
0,04\end{array}$ & $\begin{array}{l}\mathbf{0 , 2 6 *} \\
\mathbf{0 , 3 0} *\end{array}$ & $\begin{array}{c}\text { n.s. } \\
\mathbf{0 , 0 5 5}\end{array}$ \\
\hline$*$ & $\begin{array}{l}\mathrm{p}<0,05 \\
\mathrm{p}<0,005 \\
\mathrm{p}<0,0001\end{array}$ & & & & & & & \\
\hline
\end{tabular}

Note : Les banques du groupe 1 sont considérablement plus performantes que celles du groupe 2 (tableau 4). 
Les relations besoins-profit $\left(n^{\circ} 6\right)$ et besoins-performance globale $\left(n^{\circ} 7\right)$, toutes deux de type 2 , ne peuvent être interprétées qu'en examinant l'hypothèse de causalité entre ces variables. S'il est improbable que ce soient les besoins qui déterminent ces deux indicateurs de performance, il nous semble acceptable d'émettre l'hypothèse que lorsque des banquiers réalisent que leur unité n'a pas un rendement acceptable cela les pousse à être plus avides en informations sur la manière de mieux fonctionner. Étant donné la nature transversale de notre étude, il serait hasardeux et prématuré de trancher d'une manière définitive quant au sens réel de cette association.

Les relations de type 3 nous semblent être les plus intéressantes pour trois raisons. D'abord, elles ne sont significatives que pour les banques du deuxième groupe et ce, malgré la taille de ce sous-échantillon $\left(n_{2}=51\right)$. Ensuite, les banques du deuxième groupe sont celles dont la performance financière est significativement inférieure à celle du groupe 1. Finalement, c'est dans ces banques que nous avons trouvé un support modéré pour la relation congruence-performance (relations $n^{\text {os }}$ 9-11) de Galbraith (1977).

La relation sophistication-besoins $\left(n^{\circ} 8\right)$ nous amène à penser que plus les besoins en informations augmentent, plus les investissements en technologies de l'information augmentent. L'hypothèse inverse serait que ce sont justement les investissements accrus en technologies de l'information qui poussent les banquiers à exprimer davantage de besoins en informations, conscients qu'ils deviennent de la capacité de leur institution à les satisfaire.

Bizarrement, les seules relations significatives concernent les banques du premier groupe. Par contre, certaines relations sont significatives dans tout l'échantillon et dans un groupe ou dans l'autre : c'est le cas des relations de types 4 et 5 .

La relation incertitude-besoins $\left(\mathrm{n}^{\circ} 12\right)$ est significative dans le cas des banques les plus performantes. Ainsi, ces banques détermineraient leurs besoins davantage en fonction de l'incertitude environnementale qui, soit dit en passant, est perçue de la même manière par les banques les moins performantes d'après notre analyse du tableau 5. Peut-être que les banques du deuxième groupe sont plus préoccupées par leurs difficultés internes causées par une insatisfaction sur le plan de la gestion, elle-même issue de la perception qu'elles ne sont pas aussi performantes que leurs concurrentes. L'autre relation de type $4\left(n^{\circ} 13\right)$ suggère que la croissance factuelle est associée à la perception de la compétence du personnel informatique. Aucune théorie ne supporte une telle relation. De plus, les coefficients de corrélation entre les deux groupes ne présentent pas de différence statistiquement significative. 
Deux relations de type 5 révèlent que la compétence informatique perçue est significativement associée à la congruence dans le cas des banques du groupe 2. Lorsque nous comparons cette relation $\left(n^{\circ} 14\right)$ avec la relation $n^{\circ} 5$, nous pouvons avancer que dans les banques les moins performantes, la compétence du personnel informatique prime sur la sophistication technologique. Cette dernière, par contre, semble associée à la croissance perçue des banques du deuxième groupe. La différence entre les coefficients de corrélations de chaque groupe de banques est presque significative $(p<0,055)$ et mérite plus d'attention, car la question qui se pose encore ici est la suivante : est-ce que c'est la croissance perçue qui incite les banques à investir davantage dans les technologies de l'information ou est-ce les technologies de l'information qui permettent, dans certains cas, une plus grande perception de croissance?

De toutes les relations du tableau 6, une seule différencie les deux groupes à un niveau de signification inférieur à 0,05 : il s'agit de celle reliant la croissance au profit subjectif. Cette relation, prévue par la théorie de Galbraith, est plus accentuée dans les banques du deuxième groupe mais n'a pas été relevée ailleurs dans le tableau 6.

Enfin, il est intéressant de noter que malgré leurs fortes intercorrélations (tableau 3), les indicateurs de performance ne sont pas toujours associés aux mêmes variables en même temps; ce qui va à l'encontre de l'hypothèse que les indicateurs de performance subjectifs et factuels sont interchangeables.

Comme nous pouvons le voir, les relations sont extrêmement complexes, et l'interprétation de leur sens véritable du point de vue global est d'autant plus ardue qu'elles ne se manifestent pas toutes dans le même contexte.

\section{Conclusion}

Plusieurs résultats auraient pu ne pas être dévoilés n'eût été l'analyse typologique que nous avons effectuée. La théorie de Galbraith aurait pu passer pour totalement invalide dans le contexte bancaire si nous n'avions pas tenu compte de la variable dépendante de la performance financière comme critère de démarcation entre deux groupes de banques. Nous avons cependant été limité par divers aspects de notre étude.

D'abord, les contraintes imposées par une analyse transversale se sont fait sentir lors de l'interprétation de nos résultats. Quel est le sens de la causalité entre deux variables lorsqu'elles sont significativement associées? La deuxième limitation se trouve être la taille de notre échantillon de départ. Si un échantillon de 190 banques est acceptable pour une analyse traditionnelle, on devient très vite conscient que cela peut être un minimum pour une analyse 
typologique. Nous avons en effet été incapable de diviser notre échantillon en trois groupes qui soient significativement différents sur les variables critères, ce qui nous aurait permis de dégager des regroupements plus riches et des configurations d'organisations plus informatives. En outre, des hypothèses auraient pu être dégagées concernant non seulement les banques qui sont plus performantes que la moyenne, celles qui sont moins performantes que la moyenne, mais aussi celles dont la performance se situe aux alentours de la moyenne. Ce que notre analyse a montré, c'est que les chercheurs prennent un risque en considérant leurs échantillons comme représentant des entreprises homogènes et en testant des hypothèses bivariées sur ces échantillons. Le fait même qu'il puisse exister une typologie de relations telle que celle que nous avons dans le tableau 6 suggère que toutes les relations ne sont pas détectées dans une analyse exploratoire, si l'on se contente d'étudier un échantillon « homogène », si tant est que cela existe. En cela, nous pourrons bénéficier des travaux menés en psychologie par des chercheurs tels que Koffka (1963), Kohler (1964) et Wertheimer (1959) pour étudier les entreprises comme des variables, des configurations où certaines relations particulières deviennent observables, ou encore des « gestalts » qui prennent en considération les caractéristiques intrinsèques des groupements d'entreprises qui les composent. Notre étude n'a été qu'un pointeur dans cette direction.

\section{Bibliographie}

BRUNS, W.J. et F.W. MCFARLAN (1987), «Information technology puts power in control systems », Harvard Business Review, p. 89-94.

BRYNJOLFSSON, E. (1993), « The productivity paradox of information technology », Communications of the ACM, vol. $36, \mathrm{n}^{\circ} 12$, p. 67-77.

BYRNE, J.A. (1993), « The horizontal corporation : it's about managing across, not up and down », Business Week, décembre, p. 76-81.

Churchill, G.A. (1979), Marketing Research : Methodological Foundations, $2^{\mathrm{e}}$ éd., Ainsdale, Illinois, The Dryden Press.

COHEN, J. et P. CoHen (1983), Applied Multiple Regression/Correlation Analysis for the Behavioral Sciences, $2^{\mathfrak{c}}$ éd., N.J., Lawrence Erlbaum Associates.

Cohn, T. et R.A. Lindberg (1972), How Management is Different in Small Companies, New York, American Management Association inc.

CortinA, J.M. (1993), «What is coefficient alpha ? An examination of theory and applications », Journal of Applied Psychology, vol. 78, n 1, p. 98-104.

DeARden, J. (1972), «MIS is a mirage », Harvard Business Review, vol. 50, $\mathrm{n}^{\circ} 1$, p. $90-99$. 
DELONE, W.H. (1981), « Firm size and the characteristics of computer use », MIS Quarterly, vol. 5, $\mathrm{n}^{\circ} 14$, p. 65-77.

DOWNEY, H.K. et D. IRELAND (1979), «Quantitative versus qualitative : environmental assessment in organizational studies », Administrative Science Quarterly, vol. 24, $\mathrm{n}^{\circ} 14$, p. 630-637.

DRAZIN, R. et A.H. VAN DE VEN (1985). «Alternative forms of fit in contingency theory », Administrative Science Quarterly, vol. 30, $\mathrm{n}^{\circ} 4$, p. 514-539.

DUNCAN, R.B. (1972), « Characteristics of organizational environment and perceived environment uncertainty », Administrative Science Quarterly, vol. 17, p. 313-327.

FLOYD, S.W. et B. WoOldRIDGE (1990), « Path analysis of the relationship between competitive strategy, information technology, and financial performance », Journal of MIS, vol. 7, $\mathrm{n}^{\circ} 1$, p. 47-64.

FROMENT, D. (1993). «Les institutions se sont mises à l'heure de la concurrence », Les Affaires, 22 mai, p. B 1.

Galbraith, J. (1977), Organization Design, Reading, Massachusetts, AddisonWesley.

GHYMN, K.I. et W.R. KING (1976), « Design of a strategic planning management information system », Omega, The International Journal of Management Science, vol. $4, \mathrm{n}^{\circ} 5$, p. 595-607.

GlASGALl, W. et B. JAVETSKI (1993), « Shipwrecks and disasters », Business Week, 5 juillet, p. 94-95.

Gleckman, H., J. Carey, R. Mitchell, T. Smart et C. Roush (1993), «The technology payoff : a sweeping reorganization of work itself is boosting productivity, » Business Week, 14 juin, p. 57-68.

GORDON, L.A. et V.K. NARAYANAN (1984), « Management accounting systems perceived environmental uncertainty and organization structure : an empirical investigation ", Accounting, Organization and Society, vol. 9, $\mathrm{n}^{\circ}$ 1, p. 33-47.

HAMBRICK, D.C. (1981), «Environment, strategy and power within top management teams », Administrative Science Quarterly, vol. 26, $\mathrm{n}^{\circ} 2$, p. 253-276.

HAMMER, M. et J. CHAMPY (1993), « Reengineering the corporation : a manifesto for business revolution », Harper Business, New York.

HAYS, W.L. (1963), Statistics, New York, Holt, Rinehart and Winston.

HUCK, S., W. CORMIER et W. Bounds (1974), Reading Statistics and Research, New York, Harper and Row.

KoffKa, K. (1963), Principles of Gestalt Psychology, New York, Harcourt, Brace and World. 
KOHLER, W. (1964), Psychologie de la forme : introduction à de nouveaux concepts en psychologie, édition intégrale, Paris, Gallimard.

LAWRENCE, P.R. et J.W. LORSCH (1967), Organization and Environment, Boston, Massachusetts, Division of Research, Harvard Business School.

LAWRENCE, P.R. et J.W. LorsCh (1973), «A reply to Tosi, Aldag, and Storey », Administrative Science Quarterly, vol. 18, $\mathrm{n}^{\circ} 13$, p. 397-398.

O'REILly, C.A. (1980), «Individuals and information overload in organizations : is more necessarily better? », Academy of Management Journal, vol. 23, p. 684696.

PRINCE, R. (1993), « Les banques cherchent à fidéliser très jeune leur clientèle », Les Affaires, 22 mai, p. B6-B7.

RAND MCNALly BANKERS DiReCTORY (1991), dernière édition.

SMITH, K.G., C.M. GRIMM, M.J. GANNON et M. ChEN (1991), « Organizational information processing, competitive responses, and performance in the U.S. domestic airline industry », Academy of Management Journal, vol. 34, $\mathrm{n}^{\circ} 11$, p. $60-85$.

SRINIVASAN, A. (1985), "Alternative measures of systems effectiveness : associations and implications », MIS Quarterly, vol. 9, $\mathrm{n}^{\circ} 13$, p. 243-253.

STANDARD AND POOR'S REgISTER OF CORPORATIONS, DiRECTORS AND EXECUTIVES (1991), vol. 1, New York, McGraw-Hill.

Tosi, H., R. AldaG et R.G. Storey (1973a), «On the measurement of the environment : an assessment of the Lawrence and Lorsch environmental uncertainty scale », Administrative Science Quarterly, vol. 18, p. 27-36.

Tosi, H., R. Aldag et R.G. Storey (1973b), «Comment on the Lawrence and Lorsch reply », Administrative Science Quarterly, vol. 18, p. 398-400.

TUSHMAN, M.L. et D.A. NADLER (1978), « Information processing as an integrating concept in organization design », Academy of Management Review, vol. 3, $\mathrm{n}^{\circ} 3$, p. 613-624.

VENKATRAMAN, N. (1989), « The concept of fit in strategy research : toward verbal and statistical correspondence », Academy of Management Review, vol. 14, $\mathrm{n}^{\circ} 3$, p. 423-444.

WALSH, J.P. et G.R. UNGSON (1991), «Organizational memory », Academy of Management Review, vol. 16, $\mathrm{n}^{\circ} 1$, p. 57-91.

Wertheimer, M. (1959), Productive Thinking, New York, Harper.

WhISTLER, T.L. (1970), The Impact of the Computer on the Organization, New York, Praeger. 